\title{
SOBRE LA PRESENCIA DE AZOLLA FILICULOIDES LAM. EN ESPAÑA
}

\author{
E. RUIZ DE CLAVIJO, J. MUÑOZ \& A. E. SALVO
}

RESUMEN: Azolla filiculoides Lam. se cita por primera vez para España.

SUMMARY: Azolla filiculoides Lam. is reported as new to the Flora of Spain.

En el mes de Junio de 1983, durante una expedición realizada a la zona norte de la provincia de Córdoba, localizamos en aguas estancadas de los ríos Zújar y Guadamatilla, algunas poblaciones aisladas de Azolla filiculoides Lam.

Aunque en un principio consideramos que estas localidades podrían tener únicamente un interés corológico regional, puesto que Lawalrée (1964) indicaba la presencia de esta especie en España, el posterior estudio bibliográfico realizado nos hace pensar que se trata de la primera cita de este taxon para nuestro país.

El género Azolla está constituido por seis especies originarias de las regiones tropicales y templado-cálidas de América, Oceanía, Asia y Africa. Las especiales condiciones ecológicas a las que están adaptadas estas salviniales han permitido una fácil introducción antropógena de algunas especies por territorios alóctonos. Este es el caso de A. caroliniana Willd., A. pinnata R. Br. y A. filiculoides Lam., presentes en algunos paises de Europa.

Según Lawalrée (op. cit.) A. caroliniana y A. filiculoides estarian presentes en España. Sin embargo, Jalas \& Suominen (1972) omiten las citas españolas de ambas especies. Greuter, Burdet \& Long (1981) admiten la existencia en nuestro país de A. caroliniana basándose para ello en la cita de Bolós \& Masclans (1955) del Delta del Llobregat, si bien continuan en la línea de los autores del Atlas Florae Europaeae de no admitir la presencia de A. filiculoides.

A. caroliniana ha sido citada no sólo en el Delta del Llobregat (Bolós \& Masclans, op. cit.; Bolós, 1967), sino también más recientemente en la cuenca extremeña del Guadiana, concretamente en el río Guadiana a su paso por Medellín y en el río Ruecas (Perez-Chiscano, 1982).

En cuanto a la presencia de A. filiculoides existen algunos comentarios inconcretos. Así deja cierta duda la pluralización de Montserrat (1982) en la siguiente observación: "No quiero opinar 
ahora sobre las Azolla naturalizadas en el Delta del Llobregat...". Por otra parte Mathez (1980), al señalar la existencia de esta especie en Marruecos comenta: "Introduction probable à partir du Portugal ou d'Espagne...".

Las localidades donde hemos recolectado esta especie, distantes entre sí no más de 5 Kilómetros, coinciden con tramos de escasa pendiente de los ríos Zújar y Guadamatilla, de altitud comprendida entre 350 y $400 \mathrm{~m}$, y que soportan en sus riberas comunidades en las que predominan fundamentalmente Nerium oleander y Securineja buxifolia, de acuerdo con la termicidad de la zona.

Como consecuencia de la desecación estival aparecen charcas de aguas poco profundas, en la superficie de las cuales, y formando densas poblaciones de individuos en su mayoría fértiles, se ha recolectado A. filiculoides. Estas aguas estancadas acogen también comunidades de plantas sumergidas en las que predominan Potamogeton crispus, Potamogeton nodosus, Najas minor, Ceratophyllum demersum, Potamogeton pectinatus y Myriophyllum spicatum, presentando así mismo una vegetación marginal de Scirpus lacustris, Alisma lanceolatum, Eleocharis palustris, Baldellia ranunculoides, Scirpus maritimus. etc.

Posteriormente en Octubre de 1983, visitamos nuevamente la zona, localizando tan sólo algunos ejemplares estériles creciendo sobre el limo húmedo de los márgenes de estas charcas parcialmente desecadas.

\section{Material estudiado}

Córdoba: Entre Belalcázar y Cabeza de Buey: Río Zujar (UH 18), 30.VI. 1983, C. Galán \& E. Ruiz de Clavijo (COFC); Entre Belalcázar y Estación de Belalcázar: Río Guadamatilla (UH 17), 30.VI.1983, C. Galán \& E. Ruiz de Clavijo (COFC) (MGC 10340).



Mapa 1.- Localización de Azolla filiculoides en la provincia de Córdoba.

Azolla filiculoides es un taxon difícil de diferenciar de A. caroliniana, sobre todo cuando el material es estéril. Además existen bastantes discrepancias entre los autores en cuanto al establecimiento de los caracteres diferenciadores, por lo que a continua- 


\begin{tabular}{|c|c|c|}
\hline CARACTERES & A. filiculoides & A. caroliniana \\
\hline TALLOS & $\begin{array}{l}\text { Abundante y pinnada- } \\
\text { mente divididos, con } \\
\text { las ramas distantes }\end{array}$ & $\begin{array}{l}\text { Escasa y subdicotómi- } \\
\text { camente divididos, } \\
\text { con las ramas muy } \\
\text { próximas }\end{array}$ \\
\hline $\begin{array}{l}\text { HOJAS } \\
\text { (Lóbulo superior) }\end{array}$ & $\begin{array}{l}\text { Grandes (de c. } 1 \mathrm{~mm} \text { ), } \\
\text { obtusas, con el mar- } \\
\text { gen hialino ancho y } \\
\text { con papilas unicelu- } \\
\text { lares (fig. 1) }\end{array}$ & $\begin{array}{l}\text { Pequeñas (de c. } 0.5 \\
\text { mm), subagudas, con } \\
\text { el margen hialino es- } \\
\text { trecho y con papilas } \\
\text { bicelulares }\end{array}$ \\
\hline $\begin{array}{l}\text { MACROSPORAS } \\
\text { (Superficie) }\end{array}$ & $\begin{array}{l}\text { Densamente tubercula- } \\
\text { das (fig. 2) }\end{array}$ & Laxamente granulosa \\
\hline MICROSPORANGIOS & $\begin{array}{l}\text { Con 5-8 másulas (fig. } \\
\text { 3) }\end{array}$ & Con 3-6 másulas \\
\hline GLOQUIDIOS & No septados (fig. 4) & Septados \\
\hline
\end{tabular}

Azolla filiculoides.- 1: Lóbulo superior de la hoja. 2: Macrósporas. 3: Microsporangio. 4: Gloquidio (basado en MGC 10340).

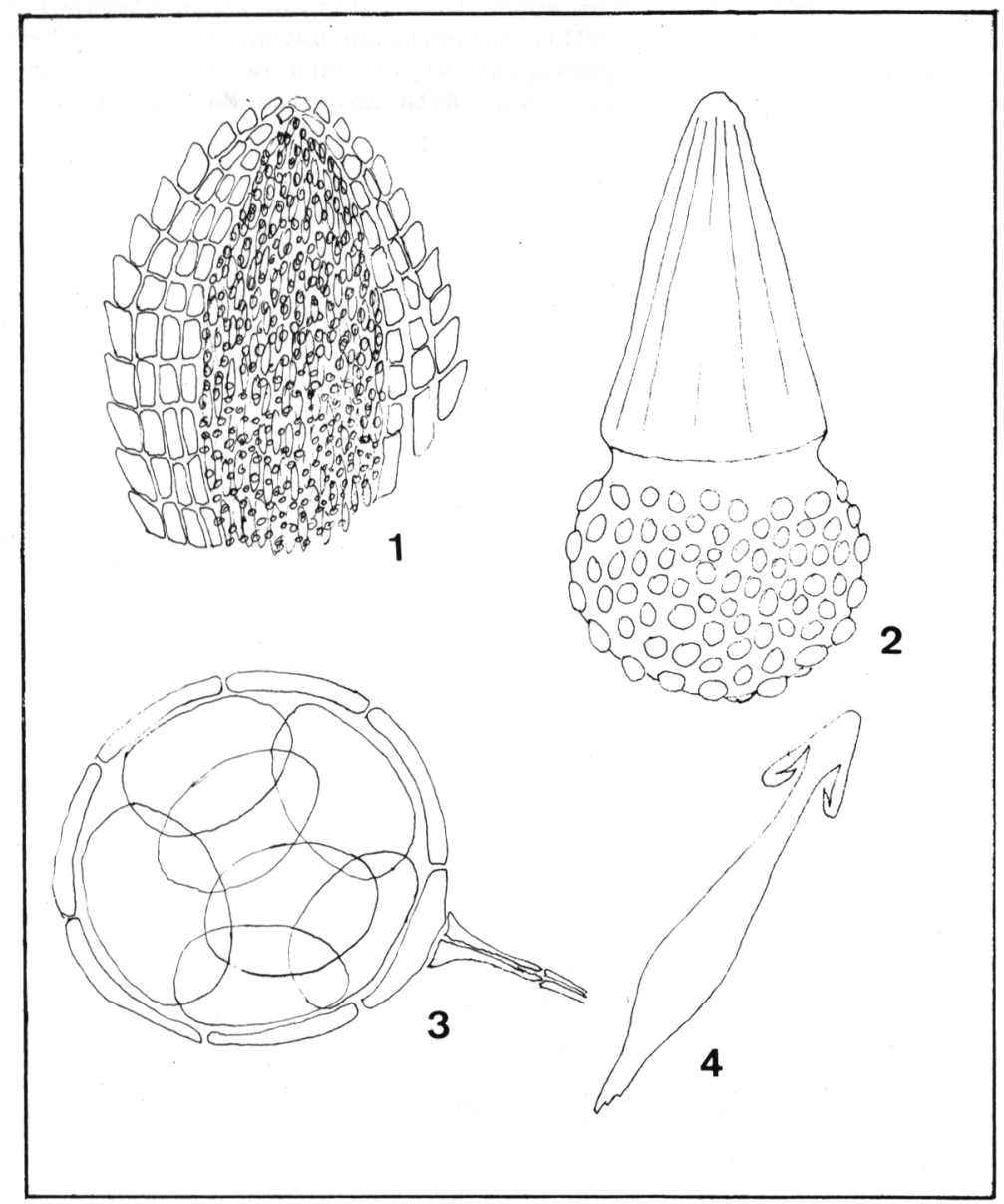


E. RUIZ DE CLAVIJO, J. MUÑOZ \& A.E. SALVO

ción presentamos un cuadro comparativo de los caracteres diagnósticos más frecuentes utilizados como diferenciadores.

\section{BIBLIOGRAFIA}

BOLOS, 0. -1967- Comunidades vegetales de las comarcas próximas al litoral situadas entre los ríos Llobregat y Segura. Mem. Real Acad. Ciencias y Artes de Barcelona, 38.

BOLOS, O. \& MASCLANS, F. -1955- La vegetación de los arrozales en la región mediterránea. Collectanea Botánica, 4(3) : 415-434.

GREUTER, W. BURDET, H.M. \& LONG, G. -1981-Med-Checklist. I. Pteridophyta. Genève \& Berlin.

JALAS, J. \& SUOMINEN, J. -1972- Atlas Florae Europaeae. 1. Pteridophyta (Psilotaceae to Azollaceae). Helsinki.

LAWALREE, A. -1964- Azolla. In Tutin, T.G. \& al. (Ed.). Flora Europaea $1: 25$. Cambridge.

MATHEZ, J. -1980-Azolla filiculoides Lam. In Greuter, W. (Ed.). MedChecklist Notulae, 1. Willdenowia, 10:15.

MONTSERRAT, P. -1982- Comentarios sobre las investigaciones pteridológicas en España, 2a parte (1981). Collectanea Botanica 13(1): 67-84.

PEREZ-CHISCANO, J.L. -1982- Aportación al estudio de los helechos de la cuenca extremeña del Guadiana. Acta Botanica Malacitana 7:193198 .

Dirección de los autores: E. RUIZ DE CLAVIJO y J. MUÑOZ, Departamento de Botánica, Facultad de Ciencias, Universidad de Córdoba, España. A.E. SALvo, Departamento de Botánica, Facultad de Ciencias, Universidad de Málaga, España. 